\title{
EchoGéo
}

28 | 2014

Police : les espaces de l'ordre, l'ordre en espace

\section{Phnom Penh ou l'ordre métropolitain : polices, pouvoirs et territoires}

Gabriel Fauveaud

\section{(2) OpenEdition}

1 Journals

Édition électronique

URL : https://journals.openedition.org/echogeo/13807

DOI : $10.4000 /$ echogeo. 13807

ISSN : 1963-1197

Éditeur

Pôle de recherche pour l'organisation et la diffusion de l'information géographique (CNRS UMR 8586)

Référence électronique

Gabriel Fauveaud, «Phnom Penh ou l'ordre métropolitain : polices, pouvoirs et territoires », EchoGéo

[En ligne], 28 | 2014, mis en ligne le 08 juillet 2014, consulté le 31 juillet 2021. URL : http://

journals.openedition.org/echogeo/13807 ; DOI : https://doi.org/10.4000/echogeo.13807

Ce document a été généré automatiquement le 31 juillet 2021.

EchoGéo est mis à disposition selon les termes de la licence Creative Commons Attribution - Pas d'Utilisation Commerciale - Pas de Modification 4.0 International (CC BY-NC-ND) 


\title{
Phnom Penh ou l'ordre métropolitain : polices, pouvoirs et territoires
}

\author{
Gabriel Fauveaud
}

\section{Introduction - La métropolisation : une mise en ordre de l'espace}

1 La " police » semble bien être absente des dictionnaires de géographie et de géographie politique (Rosière, 2008). Son rôle en tant qu'élément ou facteur de production d'espaces urbains intéresse en effet peu les géographes, alors même qu'elle représente une organisation clé de la "mise en ordre " des villes (Ocqueteau, Frenais et Varly, 2002). De même, le rôle de la police dans les processus de métropolisation apparaît peu abordé, cependant que le rapport entre espace, métropolisation et politique s'inscrit au cœur de nombreux travaux en géographie (voir notamment Claval et Sanguin, 1997 ; Prévôt-Schapira, 2001; Gervais-Lambony, 2004 ; Di Méo, 2010 ; Ghorra-Gobin, 2008 ; 2010). Afin de contribuer aux recherches récentes s'intéressant à ces thématiques, cet article veut proposer une réflexion portant sur la relation entre le fait policier et la production d'un territoire métropolitain particulier, celui de Phnom Penh, capitale du Cambodge.

2 L'évolution des modes de production des métropoles a fait l'objet de nombreux travaux critiques en géographie, qui évoquent notamment sur le rapport entre «idéologies économiques " et "formes urbaines» (Hamel, 2010), les multiples fractures sociospatiales générées par différentes formes de métropolisation (Carry et Fol, 2012), ou encore les enjeux socio-économiques liés aux phénomènes métropolitains et aux mutations des économies capitalistes et libérales (Harvey, 2010 ; 2011). Ces travaux rappellent le procès critique porté par H. Lefebvre à l'égard de l'évolution contemporaine des modes de production des villes: les espaces urbains servent des stratégies politico-économiques tout en étant la projection de rapports de production 
inégaux du système capitaliste (Lefebvre, 1972); ils seraient, en ce sens, à la fois le produit et le vecteur de l'autorité politique, qui se met en scène dans l'espace urbain tout en modelant ce dernier à son image (Lefebvre, 1974).

3 La métropolisation ${ }^{1}$ peut tout à fait faire l'objet d'un tel examen critique puisqu'elle suppose de nouveaux modes d'urbanisation du capital, parfois aussi violents qu'inégalitaires (Harvey, 2003; Appadurai, 2009). Au sein de ces processus, les forces policières jouent un rôle central, puisqu'elles permettent le maintien d'un ordre social et d'un système économique nécessaire à la production et à l'accumulation de richesses. Pour M. Foucault, la police représente ainsi un instrument étatique indispensable à la rationalisation des modes d'accumulation du capital au sein de villes toujours en expansion tant économique que démographique (Foucault, 1975, p. 251-257). Elle serait, en ce sens, une « condition d'existence de l'urbanité » (Foucault, 2004, p. 374). Cette approche se distingue notamment celle de J. Rancière qui considère la police comme un processus et une logique de distribution hiérarchique des individus en société (Rancière, 1998, p. 112). De l'appareil d'État au principe de gouvernement, la police évoque ici des significations particulièrement dissemblables: M. Foucault la considère principalement comme une institution de pouvoir lorsque J. Rancière la définit comme une stratégie de pouvoir du politique.

Cependant, une étude par les territoires du rapport entre le fait policier et le processus de métropolisation peut tout à fait se situer au croisement de ces deux approches, notamment parce que la production de territoires urbains implique des rapports de pouvoir multidimensionnels. En effet, le territoire est à la fois un produit des rapports de pouvoir et une ressource mobilisée et utilisée dans les relations de pouvoir (Raffestin, 1979; Raffestin et Butler, 2012). En ce sens, la production de territoires urbains - et métropolitains - implique doublement le fait policier. D'un côté, ce dernier peut renvoyer à la relation entre l'ordre social et l'ordre spatial, puisque la production d'un territoire procède toujours d'une « mise en ordre » des hommes et de leurs actions dans l'espace. D'un autre côté, il peut évoquer des organisations de coercition, institutionnelles ou non, ayant pour principal objectif de faire respecter des lois, codes, principes ou normes à des individus et des groupes. C'est bien au prisme de cette double dimension du fait policier - un mode de territorialisation et un instrument de territorialisation - que nous allons maintenant regarder le processus de métropolisation de Phnom Penh.

5 En situation de macrocéphalie sur son territoire national, Phnom Penh est à la fois le centre politique et économique du Cambodge. Si la capitale se situe toutefois à la marge des processus métropolitains de la région (Goldblum et Franck, 2011), l'afflux de capitaux étrangers dans la confection (Fauveaud, 2012), l'immobilier (Fauveaud, 2013) et le secteur tertiaire (Gerles, 2008) participe de son intégration à l'économie régionale et mondiale. En conséquence, les territoires de la capitale cambodgienne se transforment au rythme d'investissements privés importants, qui se traduisent par l'édification de nouveaux quartiers d'affaires au centre et de grands projets résidentiels en périphérie.

6 Cette métropolisation procède cependant d'antagonismes certains puisqu'elle s'appuie sur des modes de production de l'espace aussi inégalitaires que violents (Fauveaud, 2011 ; Springer, 2011), au sein desquels différents groupes de coercition (police, armée et milices privées) jouent des rôles de premier ordre. Les travaux critiques en économie politique sur le Cambodge présentent fréquemment ces forces comme des institutions 
étatiques défendant principalement les intérêts privés des élites politiques et économiques. Elles seraient en ce sens des instruments du maintien de l'économie néolibérale et de l'État néo-patrimonial. Par ailleurs, leur charge institutionnelle et les moyens de coercition dont elles disposent leur permettraient un accès privilégié à différentes ressources économiques (voir notamment Hughes, 2003; Kiernan et Hughes, 2007 ; Springer, 2010).

7 Derrière ces mentions de l'action policière au Cambodge se dégagent deux approches distinctes (mais non antagonistes) du fait policier. D’un côté, le rapport entre le néolibéralisme et l'action des forces de police est évoqué, entre autres, dans les travaux de D. Garland (2001), C. Tilly (2003) ou L. Wacquant $(2009 ; 2010)$ qui y voient un instrument collectif de pénalisation de la pauvreté inhérent à la néo-libéralisation de l'économie mondiale, où les intérêts privés prévalent à la mise en place d'un État social. D'un autre côté, l'accaparement de ressources fait référence à la participation de la police aux économies urbaines des "pays en développement", renvoyant ainsi aux notions d'informalité (Lautier, 2006), de corruption (Cartier-Bresson, 2000 ; Blundo et De Sardan, 2001) et, plus généralement, aux rapports de pouvoir dans la cité.

Quoi qu'il en soit, les stratégies policières au Cambodge sont généralement traitées de manière périphérique dans le but d'éclairer un problème considéré comme plus large : les évictions foncières, le contrôle de la pauvreté ou le maintien d'un système économico-politique particulier. La police est ainsi cantonnée à son rôle instrumental, évacuant quelque peu sa capacité à ordonner et à produire un territoire. Afin d'apporter un éclairage nouveau sur le rapport entre le processus de métropolisation et le fait policier, nous examinerons comment les organisations de coercition " font avec " le territoire métropolitain et comment elles participent, d'un autre côté à le produire. Deux principaux arguments seront ainsi avancés. Premièrement, la métropolisation de Phnom Penh procède de «l'ordopolis ", qui correspond à un processus de contrôle et de mise en ordre à la fois politique et économique de la capitale cambodgienne par l'intermédiaire de forces policières. Deuxièmement, la police s'insère dans les interstices de cette métropolisation particulière, en jouant notamment avec le " désordre urbain », corolaire de "l'ordopolis ».

9 Nous verrons, dans une première partie, comment le processus de métropolisation fait l'objet d'une géopolitique micro-locale particulière dans laquelle certains acteurs dominants, par l'intermédiaire de différents groupes de coercition, s'assurent un contrôle de la capitale et des modes d'accès aux ressources de la métropolisation. Dans une deuxième partie, nous regarderons comment la police suit aussi ses intérêts propres, en tirant parti de son ancrage local et des instruments de coercition dont elle dispose pour accomplir ses stratégies économiques. Nous examinerons en conclusion comment l'ordre métropolitain fait l'objet d'une récente «territorialisation réciproque » qui se traduit par une contestation, à partir de la ville-centre, de la violence métropolitaine.

Cet article s'appuie sur des données récoltées depuis 2006 à Phnom Penh. Si la police n'a pas fait l'objet d'un projet de recherche particulier, mes enquêtes de terrains auprès de ménages périurbains et avec certains membres de l'élite économique et politique m'ont permis de côtoyer et d'interroger des membres de la police et de l'armée. Mes relations avec la police ont aussi ponctué mes pratiques de la ville en tant que résident temporaire et usager quotidien du réseau viaire et des espaces publics. Certaines informations ont enfin été tirées d'entretiens réalisés depuis 2008 à la Municipalité de 
Phnom Penh et auprès d'institutions territoriales locales qui travaillent de près avec différentes forces de coercition.

\section{« L'ordopolis » ou le contrôle et la mise en ordre du processus métropolitain}

\section{Le contrôle par la périphérie}

En tant que centre politique et économique du pays, Phnom Penh a été le théâtre, depuis le début des années 1990, d'affrontements violents entre différents clans politiques se disputant le pouvoir. Aujourd'hui, la répartition autour de la capitale des contingents fidèles au parti au pouvoir représente l'aboutissement d'une stratégie de contrôle de la métropole en tant que centre politique et institutionnel du pays. «L'ordopolis » comme mode particulier de mise en ordre du processus métropolitain renvoie donc tout d'abord aux enjeux politiques du contrôle des territoires de la capitale cambodgienne.

En 1993, les premières élections générales depuis la chute du régime khmer rouge sont organisées et un gouvernement de coalition est élu. Le Funcinpec (le Front uni national pour un Cambodge indépendant, neutre, pacifique et coopératif) et le PPC (le Parti du peuple cambodgien) se partagent la direction de l'État. Les institutions internationales (Nations unies, agences de coopérations multilatérales et bilatérales), qui encadrent largement le " processus démocratique ", s'attachent à pacifier, contrôler et normaliser les forces de sécurité du pays. La création des forces armées royales du Cambodge (FARC) en 1993, de la police nationale et de la gendarmerie royale khmère (GRK) peu après les élections générales s'inscrit dans ce processus. L'objectif est notamment de créer des forces armées unifiées lorsque ces dernières sont encore largement sous la coupe de logiques partisanes. Elles se trouvent ainsi éclatées entre différents clans politiques qui se disputent le contrôle des institutions étatiques et la suprématie politique (Vichery, 1986 ; Regaud, 1992 ; Cesari, 1995 ; Findlay, 1995 ; Gottesman, 2003 ; Chandler, 2008).

Depuis 1991, les luttes pour différentes formes de domination politique ont en effet bien souvent impliqué ces forces de coercition (Um, 1993 ; Chantrabot, 2000 ; Hughes, 2003). Le Funcinpec et le PPC s'affrontent notamment par l'intermédiaire de leurs milices respectives et des brigades de police ou de l'armée qui leur sont fidèles. Le PPC et le clan du Premier ministre Hun Sen disposent cependant d'une assise beaucoup plus importante au sein des forces de coercition institutionnelles, résultat d'une stratégie engagée dès les années 1980 avec le soutien du Vietnam. L'année 1997 représente un tournant paroxysmique majeur de ces affrontements politiques, claniques et guerriers, puisque le PPC évince par un coup de force le Funcinpec du pouvoir et exerce une répression meurtrière sur les membres du camp adverse, notamment par l'intermédiaire de la brigade $n^{\circ} 70$, de la brigade de parachutistes $n^{\circ} 911$, de l'armée de gardes du corps privée de Hun Sen, d'une partie de la police nationale et de la police militaire.

Aujourd'hui, le «clan» Hun Sen, ses enfants et ses petits-enfants occupent directement, ou par l'intermédiaire de liens matrimoniaux, des postes stratégiques au sein d'institutions civiles et militaires clés (Doyle et Roeun, 2004; Marshall, 2005; Mikaelian, 2008 ; Human Rights Watch, 2012), ce qui renforce à la fois l'emprise de Hun 
Sen sur le PPC et l'emprise du PPC sur l'échiquier politico-économique cambodgien. Ce tableau se complète par des relations claniques et interpersonnelles tissées par Hun Sen qui contrôle la plupart des postes de commandement des forces de coercition. De nombreux dirigeants et hauts gradés des institutions policières civiles et militaires actuelles ont été impliqués dans les évènements de 1997, en même temps que leurs enfants occupent des postes importants au sein de ces mêmes institutions, particulièrement en province (Marshall, 2005 ; Pasquier, 2006 ; Krystel, 2009 ; Human Rights Watch, 2012)2.

15 Il n'existe pas à proprement parler de « police municipale » à Phnom Penh (Étienne, 2005 ; Un, 2009). La plupart des organisations de coercition institutionnelles sont cependant présentes dans la capitale. La police nationale, dirigée par le ministère de l'Intérieur, compte officiellement 64000 fonctionnaires répartis dans 6 principaux départements : la sécurité, le transport, l'ordre public, les frontières, l'administration et la justice. Le contingent total des FARC se situerait officiellement autour de 100000 hommes $^{3}$. La GRK est elle aussi très active dans la capitale. Cette dernière dépend du ministère de la Défense, mais possède sa propre chaine de commandement spécifique, à l'instar des autres corps de l'armée. Environ 10000 fonctionnaires composent le total des forces de la GRK dans le pays ${ }^{4}$. Cependant, le contingent précis des différentes forces policières civiles et militaires présentes dans la capitale reste inconnu.

16 Les espaces périphériques de Phnom Penh accueillent actuellement les forces policières et militaires clés du système de contrôle et de répression organisé par le PPC et le Premier ministre Hun Sen. Le maintien à proximité de la capitale des brigades $\mathrm{n}^{\circ} 70$ et $n^{\circ}$ 911, ainsi que de l'armée de gardes du corps de Hun Sen, qui jouèrent toutes trois un rôle déterminant dans les exactions commises depuis les années 1990, montre bien la stratégie d'emprise territoriale sur la capitale. À ces factions stratégiques s'ajoutent le centre de commandement des véhicules blindés de la police militaire, les différentes académies de la police et de l'armée, le quartier général de la police de la route ou encore le quartier général des FARC (cf. illustration 1).

La localisation des forces armées en périphérie de la capitale peut évoquer une stratégie géopolitique micro-locale déjà employée par le PPC lors de précédents conflits, notamment en 1997 : localiser les corps armés fidèles au régime en périphérie de la ville pour se protéger des attaques "intérieures ", pour bloquer les accès au centre politico-administratif de la cité dans le cas d'une attaque « extérieure » et pour mieux contrôler le territoire de la capitale en cas de révolte, coup d'État ou trouble social important. Un véritable "arc du contrôle armé », qui représente la dimension politico-coercitive de "l'ordopolis", se dessine alors autour de la métropole (cf. illustration 1).

\section{Le pouvoir par la marge}

18 Cependant, la capitale cambodgienne n'est pas seulement le centre politique et institutionnel du Cambodge, c'est aussi le cœur économique du pays. Différents groupes de coercition, institutionnels ou non, appuient, lorsqu'ils en ont le mandat, le processus de métropolisation, notamment en facilitant l'accumulation de capitaux pour des investisseurs privés (principalement dans l'immobilier et l'industrie) et par la mise à l'écart de populations considérées comme indésirables. Les objectifs sont d'une part, de 
maintenir la misère en dehors de la ville-spectacle, là où le tourisme se concentre et où les évènements nationaux et internationaux se tiennent ${ }^{5}$ et d'autre part, de favoriser les investissements locaux et étrangers, qui représentent les moteurs économiques $\mathrm{du}$ processus métropolitain. Ces stratégies s'inscrivent plus généralement dans une politique de «nettoyage » (Blot, 2010) ou «d'embellissement social» [beautification] (Springer, 2010) des espaces centraux de la capitale cambodgienne.

19 L'accroissement de la spéculation foncière à partir de la fin des années 1990 a notamment généré une accélération des évictions foncières de familles considérées comme installées illégalement ${ }^{6}$ sur une terre du domaine privé ou public. À Phnom Penh, près de 150000 personnes auraient ainsi été déplacées sous la contrainte (par la force ou non) depuis 1990 dans des sites de relocalisation en périphérie, parfois très lointaine, de la capitale (Sahmakum Teang Tnaut, 2011). Dans les cas d'expulsions forcées, les opérations sont menées conjointement ou distinctement par la police nationale, la police militaire, la GRK et des milices privées. Ces dernières peuvent être engagées par les promoteurs privés ou par des institutions, comme le district, la Municipalité ou le ministère de l'Aménagement du territoire (Cohre, 2009). Les gardes privés ne viennent pas seulement soutenir les forces de coercition gouvernementales, ils les remplacent parfois, disposant ainsi d'un mandat officieux octroyé par l'institution les employant ${ }^{7}$. Ce phénomène semble s'être accru au cours des trois dernières années (Worrell et Channyda, 2013).

Les périphéries urbaines deviennent marges lorsqu'elles s'apparentent à des espaces de relégation pour des populations considérées comme indésirables, voire nuisibles (Sierra et Tadié, 2008). Ces marges urbaines font partie intégrante de "l'ordopolis» puisqu'elles accueillent l'ensemble des institutions officielles du système punitif: les prisons de la police judiciaire, de Prey Sar 1 et Prey Sar 2, de Takmau et de Kampong Speu. Entre 3800 et 4000 personnes y seraient détenues dans des conditions très difficiles, avec un taux d'occupation moyen dans les 5 prisons précédemment citées s'élevant à près de $200 \%$ (Licadho, 2011). Au système carcéral officiel s'associe un dispositif de contrôle social beaucoup plus opaque : celui des " centres sociaux ", eux aussi localisés en périphérie. Officiellement, ces espaces représentent des centres d'accueil ouverts pour des personnes pauvres, principalement des toxicomanes et des délinquants, souhaitant se soigner et se réhabiliter. Officieusement, ces espaces représentent de véritables centres de détention pour les "indésirables» de la ville (Licadho, 2008), principalement des prostitués, toxicomanes, sans-abri et mendiants arrêtés dans la rue ou lors de rafles organisées par les forces de police, parfois avec l'aide du ministère des Affaires sociales. Ces lieux d'enfermement sont aussi utilisés à des fins politiques, notamment pour la mise à l'écart temporaire d'activistes et de manifestants (Chakrya, 2012). Les centres sociaux sont gérés par différents organismes comme le Comité national contre la drogue, la police militaire, la police nationale, le ministère des Affaires sociales ou la Municipalité de Phnom Penh. Près de 2000 personnes, totalement exclues du système judiciaire formel (Human Rights Watch, 2010), y seraient ainsi détenues dans l'ensemble du pays, mais majoritairement à Phnom Penh. L'action commune de différentes organisations des droits de l'Homme a permis de mettre à jour, à la fin des années 2000 , ce système de répression sociale, notamment après la mort de plusieurs détenus. Les centres de Prey Speu et de Koh Kor ${ }^{8}$ ont fait l'objet des plus importantes polémiques. 
«L'ordopolis » s'apparente ainsi à un dispositif de contrôle armé des territoires urbains et de marginalisation de certains groupes de populations, dans le but d'ordonner et de promouvoir le processus de métropolisation. Le contrôle par la périphérie et le pouvoir par la marge se posent enfin en contrepoint de la centralisation des institutions décisionnaires en matière de sécurité (ministère de la Défense, ministère de la l'Intérieur, ministère des Affaires sociales), de sanction (ministère de la Justice et différentes cours de justice) et de gestion de la capitale (notamment la Municipalité de Phnom Penh et ses différents départements) (cf. illustration 1).

Cet aspect du processus de métropolisation ne saurait cependant effacer la multiplicité des pratiques policières. En effet, les forces de coercition tirent parti de différentes formes de " désordres urbains » qui évoquent tout ce qui échappe à la métropolisation ordonnée et contrôlée. Les conflits locaux, la pratique des espaces viaires, la spéculation foncière, ou encore les activités illégales et l'économie informelle sont autant de facteurs de désordre, sur lesquels vont s'appuyer les forces policières pour tirer des bénéfices conséquents, à leur profit et au bénéfice de leurs supérieurs. Une véritable économie policière de rente se dessine alors.

Illustration 1 - L'ordre métropolitain à Phnom Penh

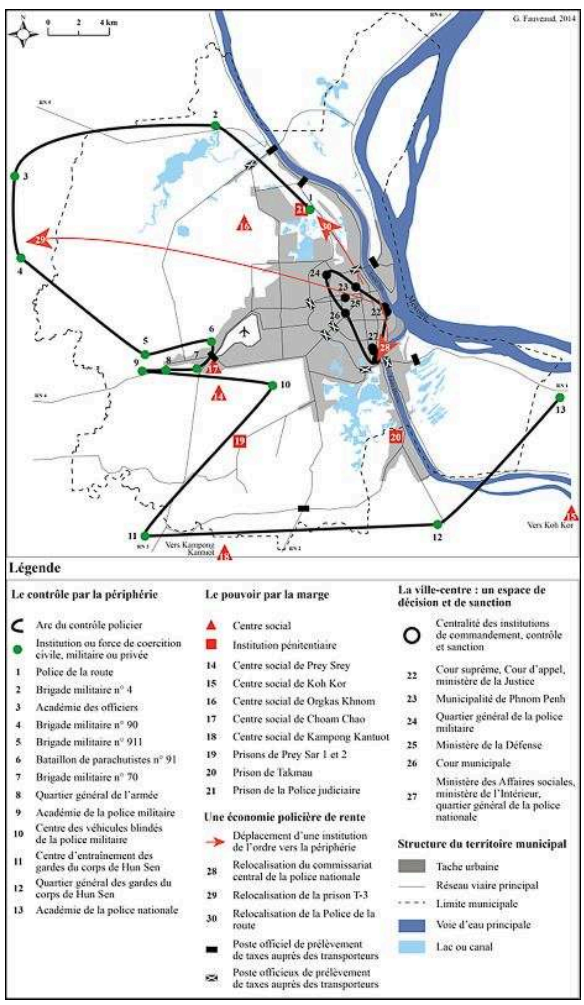

Auteur: G. Fauveaud, 2014 


\section{Tirer parti du « désordre urbain » : une économie policière de rente}

\section{Une force de coercition locale au service des dominants}

23

acheter un droit d'accès à différentes ressources et certains passe-droits (voir McDargo, 2005). Les futures positions des impétrants dans la hiérarchie policière et les lieux d'affectation sont souvent conditionnés par le montant des pots-de-vin versés aux supérieurs hiérarchiques, ainsi que par le jeu des réseaux sociaux et de l'appartenance filiale ${ }^{9}$. Parce que les charges de policier et de militaire s'achètent, les modes de contrôle des territoires urbains s'inscrivent dans une logique de captation, de partage et de redistribution de ressources financières acquises par la force ou en échange de services rendus.

Le territoire municipal de Phnom Penh est divisé en districts, eux-mêmes partagés en communes urbaines. Chaque commissariat de commune dépend du commissariat de district. La police nationale est principalement chargée d'assurer la sécurité et de gérer les conflits entre habitants sur son territoire. Le district ou les communes urbaines peuvent la solliciter mais n'ont pas d'autorité sur elle (Blunt et Turner, 2005, p. 82), car elle est à la fois autonome et soumise à l'intérêt de sa hiérarchie. Elle est par conséquent sollicitée par les habitants et les institutions locales avec précaution.

De manière générale, on évite de prévenir les forces de police ${ }^{10}$. Les échelons des communes et villages urbains sont les premiers sollicités pour régler les conflits locaux (conflits familiaux, bagarres, état d'ébriété, etc. ${ }^{11}$. Cependant, dans le cas de violences trop graves, d'un vol nécessitant une plainte, ou lorsque le conflit ne peut être résolu, la police est sollicitée. La plupart des chefs de communes urbaines et certains chefs de villages urbains sont directement connectés au poste de la police locale, par radio. Son intervention représente généralement un coût, supporté à la fois par le coupable et la victime. En effet, lorsque la culpabilité ne fait aucun doute, la police évalue et impose le paiement de «dommages et intérêts » du coupable vers la victime; à cet «acte de pouvoir » correspond généralement un paiement de la police par la victime pour s'assurer de l'application de la décision ${ }^{12}$. Cette "justice informelle», locale et immédiate, permet notamment aux belligérants de ne pas entrer dans un processus judiciaire long et coûteux dont l'issue reste incertaine (Un et So, 2012). On s'adresse ainsi localement à la police pour ne pas faire appel à la justice, pour rester « déconnecté » du système judiciaire.

Le rapport entre la population et la police n'est cependant pas égal ni homogène. Tout d'abord, lorsqu'un conflit entre deux parties implique la police, les plus riches peuvent la corrompre pour obtenir l'avantage (notamment dans le cas de conflits fonciers). Ensuite, les dispositifs d'ordre et de contrôle des territoires urbains sont bien différents en fonction des profils socio-économiques des ménages qui y habitent. Les populations les plus pauvres sont souvent plus encadrées et surveillées, étant soupçonnées de perturber l'ordre social. Elles peuvent par ailleurs être rançonnées en échange de l'occupation spontanée d'un terrain, ou pour l'exercice d'une activité informelle (comme le commerce ambulant) ${ }^{13}$. À l'inverse, les populations aux profils socioéconomiques intermédiaires peuvent pâtir de l'absence d'instances de surveillance. Il n'est pas rare que ces groupes embauchent des policiers ou des gardes de sécurité 
privés pour surveiller un espace, principalement la nuit (Fauveaud, 2013). Enfin, la présence policière n'est pas homogène sur tout le territoire métropolitain. Alors que les forces policières sont particulièrement bien visibles dans la ville-centre, les périphéries urbaines semblent, de manière générale, moins contrôlées. L'absence de forces de police ou la faible confiance que ces dernières inspirent amènent parfois les habitants à définir et à appliquer eux-mêmes des mesures coercitives envers voleurs et autres délinquants. Les journaux cambodgiens relatent régulièrement de véritables « lynchages publics » dans les périphéries de la capitale, montrant des individus ligotés et frappés, parfois à mort, par les habitants volés ou agressés ${ }^{14}$.

La police tire par ailleurs des ressources d'activités illégales, véritables corolaires de l'économie métropolitaine puisque ces dernières sont partiellement organisées par des acteurs institutionnels hauts placés, qui en tirent des bénéfices financiers conséquents. L'implication de la police et de l'armée dans le commerce d'êtres humains, la prostitution (Hoefinger, 2013) et le commerce de drogues ne fait plus de doute, même s'il paraît difficile de savoir exactement qui fait quoi. La pratique de certains lieux de prostitution par des personnages hauts placés du gouvernement empêche justement à la police d'intervenir, par peur des représailles de leur hiérarchie (Hancart-Petitet, 2010, p. 147). De même, certains chefs de gangs sont bien souvent protégés par les forces policières (voir notamment Rochigneux, 2005). Les conflits locaux et l'économie informelle et criminelle sont donc autant de désordres rentables pour les forces policières. De même, le contrôle du désordre routier et du désordre foncier représente des enjeux financiers conséquents pour les forces de coercition.

\section{Le réseau viaire et le foncier : des désordres rentables}

28 Les stratégies de contrôle policier des espaces viaires découlent de deux principales logiques distinctes et complémentaires : faciliter et contrôler le trafic (particulièrement lors des heures d'affluence); sanctionner les usagers et les taxer. La législation détermine les droits et devoirs des usagers de la trame viaire comme des autorités de contrôle. Elle se compose d'un ensemble de lois, décrets et sous-décrets votés depuis la deuxième moitié des années 2000 principalement (MPWT, 2006). Dans un souci de rentabilité, seules certains délits sont soumis à sanction, principalement le non-port du casque pour le conducteur, l'absence de rétroviseurs, l'allumage des phares en journée, le non-respect des feux de signalisation et des sens uniques, l'absence de plaques d'immatriculation et la conduite d'un véhicule disposant d'un volant à droite. Il est très rare que les usagers soient arrêtés pour d'autres infractions prévues par le code (conduite en état d'ivresse, conduite dangereuse, dépassement du nombre maximum de personnes par véhicule, etc.).

Ces tactiques légales sont associées à des stratégies territoriales. La police de la route et la GRK se placent principalement aux carrefours des grandes artères. Cependant, elles préfèrent toujours les espaces fournissant un abri pour se cacher (arbre, espace de stationnement, forme de la voirie, etc.). Les forces de police utilisent par ailleurs les aspérités et contradictions du réseau viaire pour s'assurer une plus grande « rente de situation ». Ainsi la police se trouve-t-elle à l'entrée de rues à sens unique dont le panneau signalétique est caché par un arbre ou une façade, ou au sein d'espaces donc la signalisation vient tout juste d'être modifiée. Certains espaces routiers sont alors de véritables enjeux pour les policiers : il n'est pas rare de voir des agents dormir dans des 
hamacs pour "garder » une place lucrative. Il existe donc une compétition territoriale policière, à la fois à l'échelle de la métropole entre membres de la police de la route, mais aussi entre les différents commissariats d'un même district, tout comme de véritables nœuds et axes de contrôle à partir desquels les forces de police, et plus particulièrement la police de la route et la GRK, tirent parti du désordre.

Lors d'arrestations, les contraventions sont payées directement aux policiers, souvent après négociations et entente sur le montant ${ }^{15}$. Le processus de paiement de l'amende correspond à un rituel particulier. Les policiers demandent généralement des montants plus élevés que l'amende officielle, «à la tête du client ». Ils cherchent notamment à ponctionner plus sévèrement les contrevenants qui ne sont pas aux faits des usages, ce qui vaut autant pour les étrangers que pour les provinciaux. Si les arrestations pour des pénalités mineures sont rares, un groupe de policiers en manque d'argent (notamment après les fêtes nationales) peut menacer le contrevenant d'un emprisonnement et exiger des sommes plus conséquentes.

Il est assez rare que les usagers de la route soient arrêtés pour des délits qu'ils n'ont pas commis, tout simplement parce que les infractions à la loi sont très nombreuses. Les infractions généralement sanctionnées représentent finalement une infime partie des amendes dont pourraient s'acquitter bon nombre d'usagers. Ce système de taxation s'apparente donc pour partie à un arrangement informel où l'usager comme le garant de l'application de la législation s'entendent implicitement pour élaborer une relation d'intérêt, qui se situe entre le respect de la loi, de la hiérarchie socio-institutionnelle et parfois du «bon sens ».

les usagers ne sont cependant pas égaux face à ce système informel. Les habitants les plus pauvres, qui ne peuvent payer l'amende, se font parfois saisir leurs motos. Ils doivent alors solliciter familles et amis pour payer un montant plus élevé directement au commissariat, sous peine de perdre leur véhicule. Un conducteur qui refuse de payer peut même risquer sa vie (Sovuthy, 2013). À l'inverse, les policiers n'arrêtent que très rarement les voitures arborant des plaques d'immatriculation "officielles" ou des macarons indiquant l'appartenance du conducteur à une institution civile ou militaire, illustrant ici l'intérêt de posséder une « charge » officielle de policier ou de militaire.

Le système de taxation des transporteurs de marchandises à Phnom Penh est quelque peu différent de celui des usagers car il est systématique. Officiellement, seuls les camions dont le poids est inférieur à 1,5 tonne peuvent entrer librement dans la capitale, les tonnages supérieurs devant disposer d'un laissez-passer valable pour trois mois et acheté au ministère du Transport pour 30 dollars. Officieusement, tous les camions entrant dans Phnom Penh payent pour " être tranquilles » ${ }^{16}$, le prix oscillant entre 1000 et 5000 riels par point de contrôle ${ }^{17}$. La taxation des camions se fait par la police de la route à partir de guérites installées à l'entrée de la métropole et dans le centre. Lillustration 1 montre bien que la pratique des espaces centraux est plus onéreuse, puisqu'elle suppose de passer par de plus nombreux points de contrôle, notamment officieux. À la différence des usagers donc, le paiement des transporteurs ne correspond pas à une conséquence du non-respect de la loi, mais plutôt à une contribution systématique à l'économie policière de rente.

Une partie des fonds récoltés par la police revient directement aux policiers postés sur la route (la répartition se fait en fonction des grades), lorsqu'une autre partie vient alimenter les fonds du commissariat ${ }^{18}$. Les hauts gradés du commissariat en gardent une partie tout en alimentant une caisse pour les dépenses imprévues (caisse de 
solidarité, demande inattendue des échelons supérieurs de la hiérarchie, etc.). Une partie de l'argent est enfin redistribuée au commissariat du district, qui en reversera une partie aux échelons supérieurs ${ }^{19}$.

Le haut de la hiérarchie policière profite par ailleurs des nouvelles perspectives d'enrichissement ouvertes par la croissance de la spéculation foncière depuis la fin des années 1990. De manière générale, la vente de biens fonciers et immobiliers du domaine privé de l'État à des investisseurs privés alimente les caisses des administrations propriétaires. En conséquence, une partie des institutions de l'ordre policier a ainsi été relocalisée en dehors de la ville-centre (cf. illustration 1). Par exemple, le commissariat central de Phnom Penh ainsi que l'hôpital de la police ont été déplacés (Clerc, 2005, p. 193), tout comme la police de la route relocalisée en périphérie nord. Au début des années 2000, la destruction de l'ancienne prisonT-3 en plein cœur de la ville, sa relocalisation dans la province limitrophe de Kampong Speu et la vente du terrain à la société Sokimex ${ }^{20}$ (Kyne, 2000) s'inscrivent dans le même processus, tout comme le devenir incertain de l'ancien commissariat central des autorités coloniales françaises situé en plein cœur du quartier historique (Murray, 2013).

L'abandon, la destruction et la délocalisation des anciennes et actuelles institutions pénitentiaires, judiciaires et policières sont le résultat de décisions prises au sommet de la hiérarchie politico-administrative. Si les bénéficiaires réels de ces transactions immobilières restent inconnus, il est fortement probable que la vente du patrimoine immobilier propre à chaque administration alimente à la fois les comptes personnels des hauts représentants de chaque institution, mais aussi des responsables au sein de l'État central et du PPC (voir notamment Pasquier, 2006). De l'agent de police au haut dirigeant ministériel, c'est bien une grande partie de la hiérarchie policière qui tire des bénéfices de sa charge institutionnelle. Ces stratégies de rente se situent ainsi aux interstices du formel et de l'informel, du légal et de l'illégal, de la centralité institutionnelle et de la fragmentation politique, de l'autonomie d'action et de la soumission aux supérieurs hiérarchiques, ou encore, de l'ordre et du désordre.

\section{Conclusion : " ordopolis ", " désordre urbain » et «territorialisation réciproque »}

37 L'ordre métropolitain à Phnom Penh, qui s'inscrit définitivement dans une "géographie de la violence» (Gregory et Pred, 2007), souligne finalement deux principes d'organisation, de contrôle et de mise en ordre - c'est-à-dire de production des territoires de la capitale cambodgienne. D'un côté, "l'ordopolis ", qui évoque les stratégies de contrôle politique et de mise en ordre socio-économique de la villecapitale par l'intermédiaire de différentes forces policières, véritables instruments de coercition et de violence au service d'élites politiques et économiques. D'un autre côté, le contrôle du "désordre urbain ", qui renvoie aux gains générés par la régulation des espaces viaires, par l'économie informelle et criminelle, ou encore par la spéculation foncière. En ce sens, le fait policier dans la capitale cambodgienne est à la fois un mode et un instrument de territorialisation au bénéfice d'acteurs institutionnels et privés dominants tant à l'échelle métropolitaine qu'au sein des espaces locaux, mais aussi au profit des forces de coercition elles-mêmes, qui organisent une économie policière de rente fondée tant sur le service rendu que sur la menace et la sanction. 

en ordre du territoire métropolitain, la ville-centre est devenue, depuis le début des années 2010, un nouvel espace à enjeux pour les clans au pouvoir, les forces policières et une population citadine de plus en plus contestataire. Les travailleurs de la confection $^{21}$, les victimes d'évictions foncières ${ }^{22}$ et, plus récemment, un ensemble beaucoup plus large de travailleurs précaires habitant principalement des espaces paupérisés de la capitale protestent devant certains espaces symboliques du pouvoir, comme la maison de Hun Sen en plein cœur de la ville (Pheap et Sovuthy, 2013), l'Assemblée nationale ou la Cour municipale (cf. illustration 1). De nouvelles formes de combats s'engagent alors pour l'occupation de la sphère publique et de ses espaces en même temps que les forces policières s'organisent pour contrôler et réprimer la remise en question de l'ordre métropolitain. Les groupes protestataires s'appuient notamment sur de nouvelles figures de la contestation populaire (voir notamment Chakrya et Worrell, 2013 ; Dara et Woods, 2013), comme en témoignent les pancartes montrant les photographies des «martyrs» de la répression policière brandies lors des manifestations, mais aussi sur de nouveaux médias, qui dénoncent les actions des forces policières (voir notamment Cox, 2010 et Vicheth, 2012).

La contestation politique et les espaces centraux sur lesquels elle s'appuie évoquent ainsi une "territorialisation réciproque » de l'ordre métropolitain, qui implique une centralité urbaine renouvelée, de nouvelles formes de pratiques des espaces publics et de nouvelles mesures de contrôle, de coercition et de répression leur correspondant. Il paraît ici nécessaire de ne pas considérer le rapport centre-périphérie dans sa dimension hiérarchique, mais plutôt dans sa dimension relationnelle, qui s'attacherait au système formé par ces deux types d'espaces dans le processus de métropolisation à Phnom Penh. Dans la même perspective, les marges, et notamment les lieux de détention, ne seraient pas seulement des antimondes (Morelle et Milhaud, 2006), mais aussi une condition nécessaire du monde métropolitain tel qu'il se déploie dans la capitale cambodgienne. Pouvoirs, territoires, polices, ordres et désordres entrent alors en résonnance, au point de générer des tensions parfois extrêmes, qui émergent justement lorsque la métropolisation projetée et les intérêts privés qu'elle dessert rencontrent une réalité socio-spatiale aussi multiple que désordonnée.

\section{BIBLIOGRAPHIE}

Appadurai A., 2009. Géographie de la colère : la violence à l'âge de la globalisation. Paris, Payot, 207 p.

Ascher F., 2003. « Métropolisation. In Levy J. et Lussault M. (dir.), Dictionnaire de la géographie et de l'espace des sociétés, Paris, Belin, p. 612-615.

Barton C., Sovan N., 2008. Poorhouse purgatory. The Phnom Penh Post.

Blot J., 2010. Politique de lutte contre la pauvreté ou nettoyage urbain ?: Les opérations de relogement dans la périphérie phnom-penhoise. Péninsule, nº 61, p. 221-247.

EchoGéo, 28 | 2014 
Blundo G., Olivier de Sardan J.-P., 2001. La corruption quotidienne en Afrique de l'Ouest. Politique africaine, $\mathrm{n}^{\circ} 83$, p. 8-37.

Blunt P., Turner M., 2005. Decentralisation, Democracy and Development in a Post-Conflict Society: Commune Councils in Cambodia. Public Administration and Development, vol. 25, p. 75-87.

Brunet R., Ferras R. et Théry H. (dir.), 2005. Métropole. In Brunet R., Ferras R. et Théry H. (dir.), Les mots de la géographie : dictionnaire critique, Paris, Reclus, p. 329.

Cartier-Bresson J., 2000. Corruption, libéralisation et démocratisation. Tiers-Monde, $\mathrm{n}^{\circ} 161$, p. 9-22.

Cary P., Fol S., 2012. Introduction. Géographie, économie, société, vol. 14, p. 113-126.

Cesari L., 1995. L'Indochine en guerres : 1945-1993. Paris, Belin, 315 p.

Chakrya K. H., 2012. Borei Keila families kept apart. The Phnom Penh Post.

Chakrya K. S., Worrell S., 2013. Yorm Bopha verdict upheld. The Phnom Penh Post.

Chandler D., 2008. A History of Cambodia. Chiang Mai, Silkworm Books, 365 p.

Channyda C., Redfern K., 2009. City tight-lipped on Koh Kor. The Phnom Penh Post.

Chantrabot R., 2000. Cambodge. La répétition de l'histoire (de 1991 aux élections de juillet 1998). Paris, You-Feng, $561 \mathrm{p}$.

Claval P., Sanguin A.-L., 1997. Métropoles et réalités politiques. In Claval P. et Sanguin A.-L. (dir.), Métropolisation et politique, Paris, L'Harmattan, p. 307-316.

Clerc V., 2005. Les politiques de résorption de l'habitat informel à Phnom Penh. Influence des organisations internationales et contradictions de l'action publique. Géocarrefour, vol. 80, n 3 , p. 183-196.

Cohre (Center on housing rights and eviction), 2009. Cambodia: Cohre condemns eviction of Dey Krahorm community in Phnom Penh. Phnom Penh, Cohre (communiqué de presse).

Dara M., Woods B., 2013. Tep Vanny - From Boeng Kak Protester to Globe-Trotting Advocate. The Cambodian Daily.

Di Méo G., 2010. La métropolisation. Une clé de lecture de l'organisation contemporaine des espaces géographiques. L'Information géographique, vol. 74, p. 23-38.

Doyle K., Roeun V., 2004. Securing Allegiance. Elite's Children Find Love in a Hot Political Climate. The Cambodia Daily.

Étienne P., 2005. Gestion municipale. Phnom Penh, Municipalité de Phnom Penh, 81 p.

Fauveaud G., 2011. Retour sur le drame de l'île des Diamants : l'investissement immobilier des grands projets urbains à Phnom Penh. EchoGéo, [En ligne], DOI : 10.4000/echogeo.12367.

Fauveaud G., 2012. Croissance urbaine et dynamiques socio-spatiales des territoires ouvriers à Phnom Penh. Cybergeo : European Journal of Geography, [En ligne], document 619, DOI : 10.4000/ cybergeo.25490.

Fauveaud G., 2013. Produire la ville en Asie du Sud-Est. Les stratégies socio-spatiales des acteurs immobiliers à Phnom Penh, Cambodge. Thèse de doctorat en géographie, Université de Paris 1Panthéon-Sorbonne, $496 \mathrm{p}$.

Findlay T., 1995. Cambodia, the legacy and lessons of UNTAC. SIPRI Research Report $N^{\circ} 9$, SIPRI et Oxford University Press, $238 \mathrm{p}$. 
Foucault, 1975. Surveiller et punir. Paris, Gallimard, 360 p.

Foucault, 2004. Sécurité, territoire, population. Cours au Collège de France, 1977-1978. Paris, Gallimard / Seuil, 435 p.

Garland, D., 2001. The Culture of Control, Crime and Social Order in Contemporary Society. Oxford, Oxford University Press, $307 \mathrm{p}$.

Gerles F., 2008. L'économie cambodgienne. In Forest A. (dir.), Cambodge contemporain, Paris / Bangkok, IRASEC / Les Indes Savantes, p. 189-256.

Gervais-Lambony P., 2004. Mondialisation, métropolisation et changement urbain en Afrique du Sud. Vingtième Siècle. Revue d'histoire, $n^{\circ}$ 81, p. 57-68.

Ghorra-Gobin C., 2008. Le gouvernement des "espaces métropolisés". Refonder la relation entre l'État et le territoire national. Esprit, vol. 2, p. 65-75.

Ghorra-Gobin C., 2010. De la métropolisation : un nouveau paradigme ? Quaderni, n 73, p. 25-33.

Goldblum C., Franck M., 2007. Les villes aux marges de la métropolisation en Asie du Sud-Est.

Espace géographique, vol. 36, p. 229-236.

Gottesman E. R., 2003. Cambodia after the Khmer Rouge: Inside the politics of nation building. Londres, Yale University Press, $428 \mathrm{p}$.

Gregory D. J., Pred A. R. (dir.), 2007. Violent geographies: fear, terror, and political violence. New York / Londres, Routledge, 390 p.

Hamel P., 2010. Les métropoles et la nouvelle critique urbaine. Pôle Sud, n 32, p. 153-164.

Harvey D., 2003. The New Imperialism. Oxford, Oxford University Press, 253 p.

Harvey D., 2010. Géographie et capital : vers un matérialisme historico-géographique. Paris, Syllepse, $279 \mathrm{p}$.

Harvey D., 2011. Le capitalisme contre le droit à la ville : néo-libéralisme, urbanisation, résistances. Paris, Amsterdam, $93 \mathrm{p}$.

Hancart-Petitet P., 2010. Des hôtesses de karaoké à Phnom Penh. Négociations des risques en santé de la reproduction. Moussons, $\mathrm{n}^{\circ}$ 15, p. 137-155.

Hoefinger, H., 2013. Sex, love and money in Cambodia: professional girlfriends and transactional relationships. New-York, Routledge, $214 \mathrm{p}$.

Hughes C., 2003. The political economy of Cambodia's transition, 1991-2001. New York, Routledge, $260 \mathrm{p}$.

Human Rights Watch, 2010. Off the Streets Arbitrary Detention and Other Abuses against Sex Workers in Cambodia. Phnom Penh, HRW, 76 p.

Human Rights Watch, 2012. "Tell Them That I Want to Kill Them". Two Decades of Impunity in Hun Sen's Cambodia. Phnom Penh, HRW, 68 p.

Jennar M. R., 2010. Trente ans depuis Pol Pot : Le Cambodge de 1979 à 2009. Paris, L'Harmattan, 332 p.

Kiernan B., Hughes C. (dir.), 2007. Conflict and change in Cambodia. Londres/New York, Routledge, $139 \mathrm{p}$.

Kristel M., 2009. Double rebondissement dans l'affaire du meurtre de Chea Vichea. Cambodge Post. Kyne P., 2000. Au revoir to T3: merci beaucoup pour les mémoires. The Phnom Penh Post. 
Lautier B., 2006. Discussion (suite) notes d'un sociologue sur l'usage de la notion de marge dans les sciences sociales du développement. Revue Tiers Monde, $\mathrm{n}^{\circ}$ 185, p. 17-20.

Lefebvre H., 1974. La production de l'espace. Paris, Anthropos, 485 p.

Lefebvre H., 1972. Le droit à la ville II. Espace et politique. Paris, Anthropos, 174 p.

Licadho, 2008. Illegal arrests \& Social Affairs centers: Time for Government action, not more denials. Phnom Penh, Licadho (communiqué de presse).

Licadho, 2010. Freedom of expression in Cambodia: the illusion of Democracy. Phnom Penh, Licadho, $60 \mathrm{p}$.

Licadho, 2011. Beyond Capacity 2011: A Progress Report on Cambodia's Exploding Prison Population. Phnom Penh, Licadho, $21 \mathrm{p}$.

Marchal R., 2004. Cambodge : de la guerre à la paix, ou d'un régime militaire à un régime policier. Paris, Fasopo, $54 \mathrm{p}$.

McCargo D., 2005. Cambodia: Getting Away With Authoritarianism? Journal of Democracy, vol. 16, $\mathrm{n}^{\circ} 4$, p. $98-112$.

Mikaelian G., 2008. Pour une relecture du jeu politique cambodgien : le cas du Cambodge de la reconstruction (1993-2005). In Forest A. (dir.), Cambodge contemporain, Bangkok, Irasec, p. 141-188.

Milhaud O., Morelle M., 2007. La prison entre monde et antimonde. Géographie et Cultures, $\mathrm{n}^{\circ}$ 57, p. 9-28.

MPWT (Ministry of Public Works and Transport), 2006. Law on Land Traffic. Phnom Penh, MPWT, $46 \mathrm{p}$.

Murray B., 2013. Time running out to preserve historic architecture. The Phnom Penh Post.

Ocqueteau F., Frenais J. et Varly P., 2002. Ordonner le désordre. Une contribution au débat sur les indicateurs du crime. Paris, La Documentation française, $168 \mathrm{p}$.

Pasquier S., 2006. Les basses œuvres de Hun Sen. L'Express.

Pheap A., Sovuthy K., 2013. Police Train at Freedom Park as Protesters Target Hun Sen's House. The Cambodia Daily.

Prévôt-Schapira M.-F., 2001. Buenos Aires, métropolisation et nouvel ordre politique. Hérodote, $\mathrm{n}^{\circ} 101$, p. 122-152.

Raffestin C., 1980. Pour une géographie du pouvoir. Paris, Librairies Techniques, 249 p.

Raffestin C et Butler S A, 2012. Space, territory, and territoriality. Environment and Planning D: Society and Space, $\mathrm{n}^{\circ} 30, \mathrm{p} .121-141$.

Rancière J., 1998. Aux bords du politique. Paris, Gallimard, 262 p.

Regaud N., 1992. Le Cambodge dans la tourmente : le troisième conflit indochinois, 1978-1991. Paris, L'Harmattan, $438 \mathrm{p}$.

Rochigneux G. (dir.), 2005. Cambodge soir. Chroniques sociales d'un pays au quotidien. Bangkok, Irasec, $222 \mathrm{p}$.

Rosière S., 2008. Métropole. In Rosière S. (dir.), Dictionnaire de l'espace politique. Géographie politique \& géopolitique, Paris, Armand Colin, p. 185-187. 
Sahmakum Teang Tnaut, 2011. Displaced families: Phnom Penh 1990-2011. Data on families in Phnom Penh that have been displaced either through planned relocation or forced eviction. Phnom Penh, Sahmakum Teang Tnaut, $6 \mathrm{p}$.

Sierra A., Tadié J., 2008. Introduction. Autrepart, n 45, p. 3-13.

Sovuthy K., 2013. Police Officer Shoots Man for Refusing to Pay Traffic Bribe. The Cambodia Daily. Springer S. D., 2010. Cambodia's neoliberal order: violence, authoritarianism, and the contestation of public space, Londres / New York, Routledge, 206 p.

Springer S. D., 2011. Articulated neoliberalism: the specificity of patronage, kleptocracy, and violence in Cambodia's neoliberalization. Environment and Planning A, vol. 43, p. 2554-2570.

Tilly C., 2003. The politics of collective violence, Cambridge, Cambridge University Press, 276 p.

Um K., 1994. Cambodia in 1994: The Year of Transition. Asian Survey, vol. 35, n 1, p. 76-83.

Un K., 2009. The Judicial System and Democratization in Post-Conflict Cambodia. In Öjendal J. et

Lija M. (dir.), Beyond democracy in Cambodia: political reconstruction in a post-conflict society,

Copenhague, NIAS, p. 70-100.

Un K. et So S., 2012. Cambodia's judiciary. Heading for political judicialization? In Björn D. (dir.), The Judicialization of Politics in Asia. Londres / New York, Routledge, p. 184-201.

Vickery M. T., 1986. Kampuchea: Politics, Economics and Society. Londres, Frances Pinter, 211 p.

Wacquant L., 2009. Punishing the poor: the neoliberal government of social insecurity, Durham, Duke University Press, $384 \mathrm{p}$.

Wacquant L., 2010. La tornade sécuritaire mondiale : néo-libéralisme et châtiment à l'aube du XXIe siècle. Mouvements, vol. 3, $n^{\circ}$ 63, p. 137-154.

Worrell S., Channyda C., 2013. A confronting force on streets. The Phnom Penh Post.

Site d'Interpol : http://www.interpol.int

Site de la Gendarmerie royale khmère : http://www.grk.com.kh

Site de l'Armée royale du Cambodge : http://www.army.mil.kh

Site du Parti du peuple cambodgien : http://www.cpp.org.kh

Site de la police nationale : http://www.police.gov.kh

Site du Koh Santepheap Daily : http://kohsantepheapdaily.com.kh

Site du Cambodia Express News : http://www.cen.com.kh

Cox B. (réalisateur), 2010. Who killed Chea Vichea, Cambodge, 56 minutes.

Vicheth C. (parolier), 2012. Lament of land owners. Cambodge, 6 minutes et 17 secondes.

\section{NOTES}

1. La métropolisation peut se définir comme un processus par lequel une ville accroît son influence nationale, régionale et mondiale et sa participation à l'économie globale par l'accumulation de fonctions de centralités (politiques, économiques, culturelles, etc.) et par l'accroissement de son attractivité, notamment démographique (Asher, 2003 ; Brunet, Ferras et Théry, 2005 ; Rosière, 2008). 
2. L'actuel ministre de la Défense, le Général Tea Banh (dont les enfants sont placés à des postes importants de la hiérarchie militaire), a largement été impliqué dans le coup de force de 1997, tout comme le chef actuel de la GRK, le commandant Sao Sokha, qui occupe ce poste depuis 1993. Huy Piseth, aujourd'hui Lieutenant Général et sous-secrétaire d'État à la défense, commandait justement la brigade $n^{\circ} 70$ au moment des évènements de 1997. Chap Pheakdei, lui aussi proche de Hun Sen, dirige la brigade $n^{\circ} 911$. Enfin, l'actuel commandant en chef de la police nationale, Neth Savoeun, est marié à une sœur de Hun Sen. Sans surprise, tous les personnages précédemment cités, à l'exception de Huy Piseth, siègent au comité exécutif du PPC.

3. http://www.army.mil.kh

4. http://www.grk.com.kh

5. Les opérations de nettoyage s'intensifient ainsi logiquement lors d'évènements particuliers (Human Rights Watch, 2010).

6. La titrisation foncière formelle n'étant pas la norme à Phnom Penh, les conflits fonciers sont récurrents et bien souvent soumis à la raison du plus fort.

7. Par exemple, en janvier 2012, une trentaine de personnes, qui protestaient contre leur éviction forcée d'un terrain qu'elles occupaient dans la ville-centre, ont été arrêtées lors d'une manifestation devant la Municipalité de Phnom Penh par des gardes de sécurité employés par le district de Daun Penh. Les protestataires ont été envoyés dans le Centre social de Prey Speu, sans que la détention fasse l'objet d'une décision de cour ou d'un mandat officiel.

8. Fermé en 2008 et officiellement en réhabilitation depuis lors, Koh Kor est un ancien centre de détention et d'exécution khmer rouge situé sur une île du fleuve Bassac non loin de Phnom Penh. Sa découverte a fait grand bruit (Barton et Sovan, 2008 ; Channyda and Redfern, 2009).

9. Entretien avec un membre de la police nationale le 15 juin 2009.

10. Enquêtes de terrain entre 2008 et 2012.

11. Enquêtes dans des communes périurbaines entre 2008 et 2009.

12. Entretien avec un membre de la police nationale le 8 juillet 2009.

13. Enquêtes dans des communes périurbaines entre 2009 et 2012.

14. Voir notamment les journaux en ligne Koh Santepheap Daily ( http:// kohsantepheapdaily.com.kh ) et Cambodia Express News (http://www.cen.com.kh ).

15. Le montant demandé aux Cambodgiens pour des "pénalités mineures » est généralement compris entre 3000 et 5000 riels (entre 0,50 et 1 euro environ) pour les motos et entre 5000 et 10000 riels (entre 1 et 2 euros environ) pour les voitures.

16. Entretien le 25 novembre 2013 avec le représentant d'une société de transport nationale basée à Phnom Penh.

17. Entre 20 centimes d'euro et 1 euro environ.

18. Entretien le 25 novembre 2013 avec le représentant d'une société de transport nationale basée à Phnom Penh.

19. Entretien avec un lieutenant de police le 15 octobre 2009.

20. La prison T-3, construite par les Français en 1916, a accueilli des prisonniers jusqu'à la fin des années 1990. Sokimex, la plus importante compagnie pétrolière cambodgienne, est dirigée par Sok Kong, un puissant entrepreneur membre du PPC. L'entreprise possède de multiples filiales et a réalisé de nombreux investissements immobiliers dans la capitale depuis la deuxième moitié des années 1990, notamment dans l'hôtellerie. Cette compagnie serait une source de financements majeure du PPC.

21. Entre novembre 2013 et janvier 2014, plusieurs habitants ont été tués par la police à Phnom Penh lors de manifestations ouvrières en périphérie de la capitale.

22. En 2011, une manifestation organisée par le Cambodia Peace Network a rassemblé dans la ville-centre plus de 300 « communautés » victimes d'évictions venues de tout le pays (Licadho, 2010). 


\section{RÉSUMÉS}

Cet article propose une analyse critique du processus de métropolisation de Phnom Penh au prisme des pratiques et stratégies policières. J'avancerai tout d'abord que le processus de métropolisation procède d'un mode particulier de contrôle et de mise en ordre politique et économique des territoires de la capitale, «l'ordopolis ». Je postulerai ensuite que les stratégies des forces policières s'insèrent dans les interstices de la métropolisation en s'appuyant sur différentes formes de "désordres urbains ", qui nourrissent une économie policière de rente. Nous verrons, en conclusion, que l'ordre métropolitain fait l'objet d'une récente «territorialisation réciproque », qui se traduit par une centralité urbaine renouvelée.

In this article, I propose a critical analysis of the Phnom Penh metropolization process by investigating practices and strategies of local police forces. Firstly, I argue that the metropolization process is based on a particular political and economic ordering of the city's territories, the "ordopolis". Secondly, I argue that strategies and practices of local police forces rely on different forms of "urban disorders", which support a rent-seeking police economy. In conclusion, we will see that the metropolitan order has been recently the subject of a "reciprocal territorialization", which means a renewal of the urban centrality.

\section{INDEX}

Keywords : Phnom Penh, power, metropolization, police, territory

Mots-clés : Phnom Penh, pouvoir, métropolisation, police, territoire

\section{AUTEUR}

\section{GABRIEL FAUVEAUD}

Gabriel Fauveaud, gabriel.fauveaud@gmail.com, est chercheur post-doctorant au Centre d'études et de recherches internationales de l'Université de Montréal (Cérium) et à l'observatoire Ivanhoé Cambridge du développement urbain et immobilier de la faculté d'aménagement de l'Université de Montréal. Il a publié récemment :

- Fauveaud G., 2012. À la recherche des savoirs urbains, les enjeux socio-spatiaux du marché du logement à Phnom Penh. Péninsule, nº 64, p. 123-154.

- Fauveaud G., 2012. Croissance urbaine et dynamiques socio-spatiales des territoires ouvriers à Phnom Penh. Cybergeo : European Journal of Geography, [En ligne], article 619, mis en ligne le 23 août 2012.

- Fauveaud G., 2011. Retour sur le drame de l'île des Diamants : l'investissement immobilier des grands projets urbains à Phnom Penh. EchoGéo, [En ligne], Sur le Vif, mis en ligne le 3 mai 2011. 